\title{
LOS JUEGOS ELEGIDOS POR NIÑOS EN EL COLEGIO, EN LA CASA Y EL FAVORITO
}

\author{
María Guadalupe de los Milagros Damián-Díaz \\ UNAM, FES IZTACALA
}

MÉXICO

\begin{abstract}
RESUMEN
El juego fomenta el desarrollo de la creatividad, la creación de nuevas habilidades y ha sido aprovechado como recurso pedagógico. Este estudio cuantitativo-exploratorio tiene por objetivo describir las preferencias de los juegos de los niños de primaria en diferentes espacios, escuela, casa y el favorito. Se aplicaron cuestionarios a 2,167 niños y niñas de primero, segundo y tercero de primaria, de escuelas públicas y privadas. Se organizaron los datos y se llevó a cabo un análisis cuantitativo de los datos. Los datos muestran que en el colegio los niños y niñas juegan con mayor frecuencia atrapadas en los tres grados, En la casa juegan muñecas las niñas del primer grado, y futbol los niños segundo y tercer grado. Y por último en el juego favorito, en el primer grado los niños juegan futbol y las niñas muñecas, en el segundo y tercer grado los niños juegan futbol y las niñas escondidas. Este estudio exploratorio puede ser una opción importante para diseñar un sistema de enseñanza aprendizaje basado en las elecciones de los juegos de los niños y niñas mexicanas en estos grados para motivarlos a participar de forma más activa y mejorar su aprendizaje.
\end{abstract}

Palabras Clave:

Juego, colegio, casa, juego favorito, niños escolares, mexicanos

\section{THE GAME CHOOSEN BY PREESCOLAR CHILDREN AT SCHOOL, HOME AND FAVORITE ONE}

\begin{abstract}
The game promotes the development of creativity, creating new skills and was been used as an educational resource. This quantitative exploratory study aims to describe the preferences of the games primary school children in different spaces, school, home and favorite. Questionnaires were applied data to 2,167 children in first, second and third grade, public and private schools. were organized and performed a quantitative analysis of data. The data show that in school the children play more often trapped in the three grades, in the house girls play dolls first grade, and football the second and third grade children. Finally, in the favorite game in the first grade boys and girls play soccer dolls, in the second and third grade children play football and hidden girls. This exploratory study may be important to design a system of learning based on the choices of children's games and Mexican girls in these grades to motivate them to participate more actively and improve their learning option.
\end{abstract}

Keywords:

Play, school, home, favorite game, school children

Bitácora del Artículo:

| Recibido: 2 de Agosto de 2015 | Aceptado: 2 de Septiembre de 2016 | Publicado en línea: Enero-Junio de 2016 | 


\title{
LOS JUEGOS ELEGIDOS POR NIÑOS EN EL COLEGIO, EN LA CASA Y EL FAVORITO
}

\author{
María Guadalupe Milagros Damián-Díaz
}

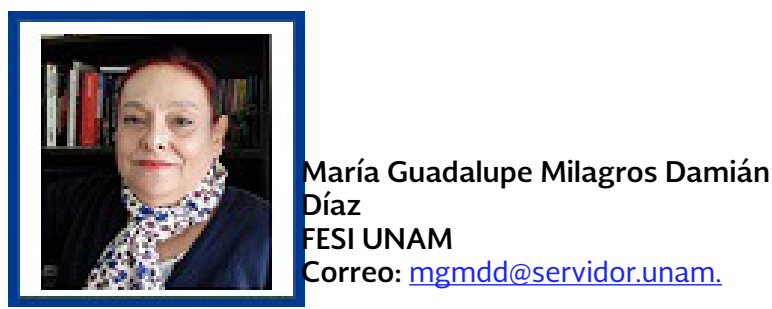

Dra. en Desarrollo Psicológico y Aprendizaje Escolar por la Universidad Autónoma de Madrid, España. Docente en el programa de Licenciatura en Psicología de la FESI, UNAM, así como en el programa de Maestía de Modificación de Conducta, UNAM.

\section{CONTRIBUCIÓN DE LA AUTORA}

Se atribuye a la autora la responsabilidad única sobre el trabajar al concibir, desarrollar y coordinar el proyecto de investigación.

\section{AgRADECIMIENTOS}

Agradezco a todas aquellas personas que participaron e hicieron posible la realización de este estudio.

\section{DATOS DE Filiación DE LA Autora}

Profesor titular FES Iztacala, UNAM

\section{Copyright: (C) 2016 Damián-Díaz, M. G. M.}

Este es un artículo de acceso abierto distribuido bajo los términos de la licencia Creative Commons Reconocimiento-NoComercial 4.0 Internacional, por lo que su contenido gráfico y escrito se puede compartir, copiar y redistribuir total o parcialmente sin necesidad de permiso expreso de su autor con la única condición de que no se puede usar con fines directamente comerciales y los términos legales de cualquier trabajo derivado deben ser los mismos que se expresan en la presente declaración. La única condición es que se cite la fuente con referencia a la Revista Digital Internacional de Psicología y Ciencia Social y a su autor. 


\section{TABLA DE CONTENIDO}

INTRODUCCIÓN

La importancia del juego en el Desarrollo Psicológico y en los procesos de aprendizaje-enseñanza, 4

Las emociones y el juego, 5

MÉTODO

Materiales , 6

Procedimiento, 6

DiCUSIÓN Y CONCLUSIONES 


\section{INTRODUCCIÓN}

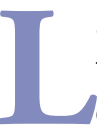

a importancia de estudiar el juego radica en la fuerte influencia y en la relación directa que tiene en el desarrollo psicológico, en el aprendizaje y en los aspectos emocionales y sociales de los niños y niñas. Se ha demostrado que el juego amplía la gama de experiencias, a través de éste se fomenta el desarrollo de la creatividad, la creación de nuevas habilidades que permiten a los niños participar en el proceso de toma de decisiones para establecer y codificar las reglas del juego en el grupo; $y$, sobre todo, el juego ha sido aprovechado como recurso pedagógico (Delval, 1991) y como técnica terapéutica en la psicoterapia infantil (Hugges, 2006; D'Agostino \& Raimbalult, 2004).

Hay autores como Tejerina (1999) que concibe al juego como un lugar de libertad, el ambiente propicio para el descubrimiento y el hallazgo. Reconoce su gran potencial pedagógico como un impulso eficaz para que los niños y adultos se expresen, esto significa la oportunidad para dar paso a la actividad creadora. Para Bernabeu y Goldstein (2009) el juego promueve y facilita cualquier aprendizaje sea éste de tipo físico y psicológico. Así mismo, describen las ventajas de las actividades lúdicas en el contexto educativo, las cuales se refieren a que: facilitan la adquisición de conocimientos, aumentan la motivación, fomentan la cohesión del grupo y la solidaridad, así como también, favorecen el desarrollo de la creatividad, la percepción e incrementan el autoconcepto, y, por último, permiten a través de la educación influir en valores como son la tolerancia, el respeto, la responsabilidad y la libertad.

Huizinga (1970) enfatiza que el juego es más antiguo que la cultura, lo cual presupone una sociedad humana, inclusive, menciona que los animales también juegan desde pequeños.

Es reconocido el hecho de que Piaget (1992) considera al juego como expresión y manifestación del pensamiento infantil. El juego para él es una manifestación cognitiva del niño, construida espontáneamente por el pequeño para enfrentar la realidad a la cual debe adaptarse. Piaget explica que los niños al estar jugando, elaboran y desarrollan sus propias estructuras mentales. Desde esta perspectiva, para el autor el juego consiste en una orientación del propio niño hacia su comportamiento, es una preferencia de los medios sobre los fines de la conducta.
Vygotsky (1979) concibe al juego, y especialmente al simbólico, como un factor básico y transcendental para el desarrollo del niño, ya que el mayor autocontrol del niño se produce en el juego, porque crea una zona de desarrollo próximo en el niño, durante la cual, está siempre por encima de su edad promedio.

Puede decirse que los teóricos están de acuerdo en la necesidad absoluta de jugar para lograr un desarrollo psicológico sano, por lo que las características del juego pueden resumirse en los siguientes conceptos: a) El juego es absorbente, b) Es organizador, c) Es transformador de la realidad y d) Permite a los niños hacer una variación de la realidad.

\section{La importancia del juego en el Desarrollo Psicológico y en los procesos de aprendizaje- enseñanza}

Piaget (1992), encauzado por sus intereses epistemológicos, estudió los procesos de simbolización en los individuos y encontró en el juego, principalmente en el simbólico, una oportunidad para explicar el paulatino abandono de las formas egocéntricas de pensamiento y la progresiva construcción de modalidades lógicas cada vez más avanzadas.

Piaget (1995) y Bruner (1989) consideran que el juego constituye un medio primordial para estructurar el lenguaje y el pensamiento crítico y creativo. Cuando el niño participa en situaciones lúdicas se favorece el paso de la inteligencia práctica a la representativa, posibilita el despliegue de la imaginación creadora y de la acción transformadora por parte del niño. La actividad lúdica resulta ser el motor del pensamiento y de la razón, tiene la propiedad de ser una forma de actividad poderosa que fomenta la vida social y constructiva del niño (Piaget, 1979, como se citó en Aizencang, 2005). Mientras que el juego promueve la generación de nuevas formas psicológicas y estimula a reflexionar sobre los procesos cognoscitivos del sujeto.

Piaget (1995) enfatiza que cualquier adaptación verdadera al medio supone una imitación, la cual participa en la incorporación de comportamientos que obligan a modificar las propias estructuras cognoscitivas del niño; en el juego se distorsiona la realidad externa a favor de la integridad de las propias estructuras mentales del niño.

Piaget declara que existen tres tipos de estructuras que caracterizan los juegos infantiles y que suponen el criterio a la hora de clasificarlos: el ejercicio, el símbolo y la regla. El juego de ejercicio es el primero en aparecer y abarca las actividades iniciales que el niño realiza con su cuerpo. Son los llamados juegos sensoriomotores que se caracterizan por la ausencia de símbolos y reglas, y que en algunos aspectos se pueden considerar similares a la conducta animal. 
De igual manera, Piaget vincula la capacidad de jugar con la capacidad de representar o de simbolizar. Se puede afirmar que le concedía un papel muy relevante, sobre todo al juego simbólico, que no es sólo asimilación de lo real al yo, como el juego en general, sino asimilación consolidada por un lenguaje simbólico construido por el yo y modificable a la medida de las necesidades de cada niño.

El juego simbólico aparece en los niños hacia los dos años aproximadamente y requiere la representación de un objeto ausente, su función principal es la realización de deseos y la resolución de conflictos, y esto supone un cambio cualitativo muy importante en comparación con el placer que produce el ejercicio sensoriomotor.

El juego de reglas implica relaciones sociales y una regularidad pactada por el grupo cuya violación es considerada como una falta. Piaget considera que los tres tipos de juego corresponden a las estructuras de la génesis de la inteligencia (sensoriomotora, representativa y reflexiva).

Lo anterior fue importante considerarlo cuando estudiamos los juegos, estos tienen propósitos diferentes, así, el juego físico proporciona la oportunidad de realizar un ejercicio muy importante para el desarrollo esquelético y muscular, el juego social ofrece un marco para el aprendizaje de las relaciones sociales y la resolución de conflictos, principalmente cuando va acompañado de la necesidad de tener que respetar ciertas reglas pactadas entre los participantes.

Otro autor que aporta información relevante sobre el desarrollo psicológico y el juego es Vygotsky que considera al juego como un factor central del aprendizaje y generador de la adaptación social, dice textualmente, "todas las funciones psicointelectivas superiores aparecen dos veces en el curso del desarrollo del niño: la primera vez en las actividades colectivas, en las actividades sociales, o sea, como funciones intrapsíquicas; la segunda vez, en las actividades individuales, como propiedades internas del pensamiento del niño, o sea, como funciones intrapsíquicas" (Vygotsky 1979, p. 156).

Precisamente, esta idea de Vygotsky de que el juego, sobre todo el socio-dramático, contribuye en gran medida al análisis del juego en la escuela, éste representa una herramienta metodológica para el trabajo con niños, durante el juego el niño incrementa su potencial cognoscitivo, de allí que pueda considerarse un dispositivo para explotar el potencial real del niño en lo referente a la toma de conciencia, racionalización, interiorización de normas y nivel de significación (Elkonin, 1980).

También el juego dramático es útil como estrategia didáctica para desarrollar el pensamiento creativo en alumnos de diferentes niveles educativos, apoyado en la teoría interactiva y psicosocial de la creatividad (De la Torre, 2006).

Lo que postula Vygotsky (1970) acerca de que la sociabilidad del niño es el punto de partida de sus interacciones sociales con el medio ambiente sociocultural que lo rodea. En este sentido es necesario comprender que "por origen y por naturaleza el ser humano no puede existir ni experimentar el desarrollo propio de su especie de una manera aislada; tiene necesariamente su prolongación en los demás; de modo aislado no es un ser completo" (p. 341).

Por todo lo anterior, el juego es una valiosa herramienta en el proceso de enseñanza, aprendizaje y en la socialización de los niños y niñas porque permite crear ambientes de recreación que predisponen y motivan, y que a la vez son fuente de aprendizajes significativos en la vida social y cultural en su entorno natural (Gómez, 1991).

\section{Las emociones y el juego}

A través de los juegos los niños van confrontando su mundo interno con la realidad externa, permitiéndoles modificar situaciones que pueden resultarles no placenteras, tal como "tener que ir al médico" y luego jugar al "médico", el niño juega para confrontar y cambiar la angustia que esa situación le había provocado; el jugar le proporciona una salida al miedo que sentía.

Hay juegos que se parecen a los sueños y tal como en estos, aquellos proporcionan una salida a la angustia y expresan un deseo inconsciente. De esta forma los juegos permiten al niño irse apropiando de la realidad que lo rodea bajo la forma cultural vigente y se adecua a ésta, a través de las normas de convivencia oportunas para fortalecer sus sentimientos de seguridad y pertenencia (West, 2000).

Para Ramírez (2004) las emociones tienen tres componentes: la cognición o pensamiento, que se refiere a los juicios y pensamientos que surgen de un estado concreto o situación. El segundo hace referencia a la experiencia, es algo vivencial que se puede sentir en el cuerpo, esto es, las reacciones fisiológicas que se manifiestan a partir de un sentimiento propiciado por un evento externo, y finalmente, el tercero es la reacción expresiva, como son los movimientos corporales que se realizan con un gesto, tono de voz, movimiento o reacción física-motora.

Surge de la necesidad de conocer y expresar las emociones principales, por lo que es aconsejable que los niños deben ponerse en contacto con aquel juego que les permita buscar la vía para incrementar o decrementar la magnitud de sus emociones, ya sean estas placenteras o no placenteras, lo que los lleva a distinguir los juegos acordes a sus necesidades psicológicas vigentes, su sexo y la permisividad que el entorno social le va marcando culturalmente (Bisquerra, 2000). 
En el mismo sentido, Winnicott (1981) considera que el niño juega para expresar sus sentimientos, para controlar la ansiedad y establecer contactos sociales e integrar su personalidad; además, defiende la idea de que el juego es por sí mismo terapéutico tanto para el niño como para el adulto, sin perder de vista que al jugar se tiene toda la libertad de crear. El autor también fortaleció la idea de que el juego libre es inevitable para un desarrollo emocional normal y estable, y la necesidad imperiosa de un juego de rol social e individual libre, para desarrollar una identidad humana.

El niño mediante el juego se va apropiando de valores, reglas y comportamientos socialmente aceptados, desde esta perspectiva el juego es característico de los niños y constituye una reconstrucción social, conjuntamente con las interacciones de los adultos, y comprendidas por los niños, aunque sea de modo parcial. (Freud, 1925, como se citó en Garzarelli, 2005).

A través del juego le permite al niño ejercer sus posibilidades físicas y psicológicas, competir con otros niños, construir su inteligencia y consolidar su personalidad. El juego contribuye al pleno desarrollo, respetando el carácter global de la personalidad en el niño.

El Objetivo de este estudio exploratorio-cuantitativo es identificar, describir y analizar la elección de los juegos que practican los niños de primero, segundo y tercer grado de primaria, de escuelas oficiales y privadas, en contextos como son: el colegio, el barrio o casa, y el juego favorito, con el propósito de comparar si existen diferencias en la elección de los juegos.

Preguntas de investigación:

- ¿Existen diferencias entre niños y niñas por los grados (primero, segundo y tercero de primaria), de escuelas públicas y privadas, en la elección de los juegos en el colegio?

- ¿Existen diferencias entre niños y niñas por grados (primero, segundo y tercero de primaria), de escuelas públicas y privadas, en la elección de los juegos en la casa o barrio?

- ¿Existen diferencias entre niños y niñas por grados (primero, segundo y tercero de primaria), de escuelas públicas y privadas, por la elección de los juegos favoritos?

\section{Método}

\section{Sujetos:}

2,167 niños y niñas de primero, segundo y tercero de primaria, de edades comprendidas entre los 5 a 13 años, tanto de escuelas oficiales como privadas (ver tabla1).
Tabla 1.

Muestra seleccionada de niños de primaria inferior.

\begin{tabular}{|c|c|c|c|c|}
\hline \multicolumn{2}{|c|}{ GRADO ESCOLAR } & \multicolumn{2}{|c|}{ TIPO DE ESCUELA } & \multirow[t]{2}{*}{ TOTAL } \\
\hline & & Pública & Privada & \\
\hline \multirow{3}{*}{$1^{\circ}$ primaria } & Masculino & 157 & 131 & 288 \\
\hline & Femenino & 191 & 114 & 305 \\
\hline & Total & 348 & 245 & 593 \\
\hline \multirow{3}{*}{$2^{\circ}$ primaria } & Masculino & 231 & 171 & 402 \\
\hline & Femenino & 209 & 175 & 384 \\
\hline & Total & 440 & 346 & 786 \\
\hline \multirow{3}{*}{$3^{\circ}$ primaria } & Masculino & 198 & 193 & 391 \\
\hline & Femenino & 224 & 173 & 397 \\
\hline & Total & 422 & 366 & 788 \\
\hline \multirow{3}{*}{ Total } & Masculino & 586 & 495 & 1081 \\
\hline & Femenino & 624 & 462 & 1086 \\
\hline & Total & 1210 & 957 & 2167 \\
\hline
\end{tabular}

\section{Materiales}

\section{Aparatos y materiales}

Cuestionarios impresos, computadora y Programa estadístico SPSS, grabadora, mesas y sillas, lápices y colores.

\section{Variables Atributivas}

Sexo, grados escolares y las escuelas públicas y privadas.

\section{Variables Dependientes:}

El Juego que se practica en la escuela, el Juego que se practica en el barrio o en la casa y el Juego favorito.

\section{Procedimiento}

\section{Primera fase:}

Aplicación del cuestionario en las escuelas que lo permitieron y ante la presencia de la maestra o maestro. El cuestionario contempla lo siguiente:

1. Datos personales como son: edad, sexo, tipo de escuela, grado escolar

2. Las siguientes preguntas abiertas:

- ¿Qué juegas con tus amigos en la escuela?

- ¿Qué juegas con tus amigos en tu casa?

- ¿Cuál es el juego que más te gusta y cómo se juega?

Las instrucciones que se les dieron a los niños fueron claras y detalladas: 
- "Por favor, contesten este cuestionario, nos interesan sus respuestas. Traten de contestarlo con la verdad y lo mejor que puedan".

- "Los niños que necesiten ayuda para comprender las preguntas por favor pídanmela".

- "No hay respuestas correctas ni incorrectas sólo verdaderas".

\section{Segunda fase:}

Se organizó la información en función de las variables estudiadas.

\section{Tercera fase:}

Análisis cuantitativo de los datos, se organizaron los datos en función de las variables para la descripción de estos; se utilizó el estadístico descriptivo y no paramétrico Crosstab para ordenar los datos. Se obtuvieron los puntajes de los niños en términos de las más altas frecuencias en su género y por los contextos en que se desarroIlaron los juegos: el juego que practicaban en el colegio, en casa o en el barrio, y por su juego favorito.

\section{Resultados}

Se presentan los datos en frecuencia por la gran diversidad de juegos que los niños describieron y los de más alta frecuencia se seleccionaron según niños y niñas en las diferentes variables atributivas; $y$ entre los juegos que eligen los niños y las niñas en los diferentes contextos.

En el colegio los niños y niñas juegan con mayor frecuencia atrapadas en los tres grados (ver figura 1)

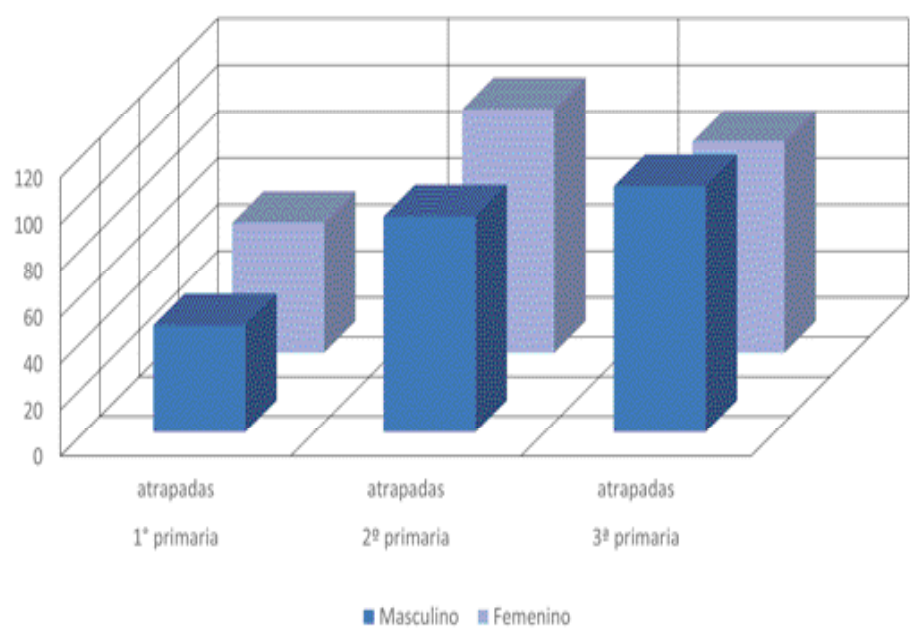

Figura 1.

Frecuencias de juego en el colegio de niños y niñas

En el barrio o casa juegan muñecas las niñas del primer grado y futbol los niños de $2^{\circ}$ y $3^{\circ}$ grado (ver figura 2 ).

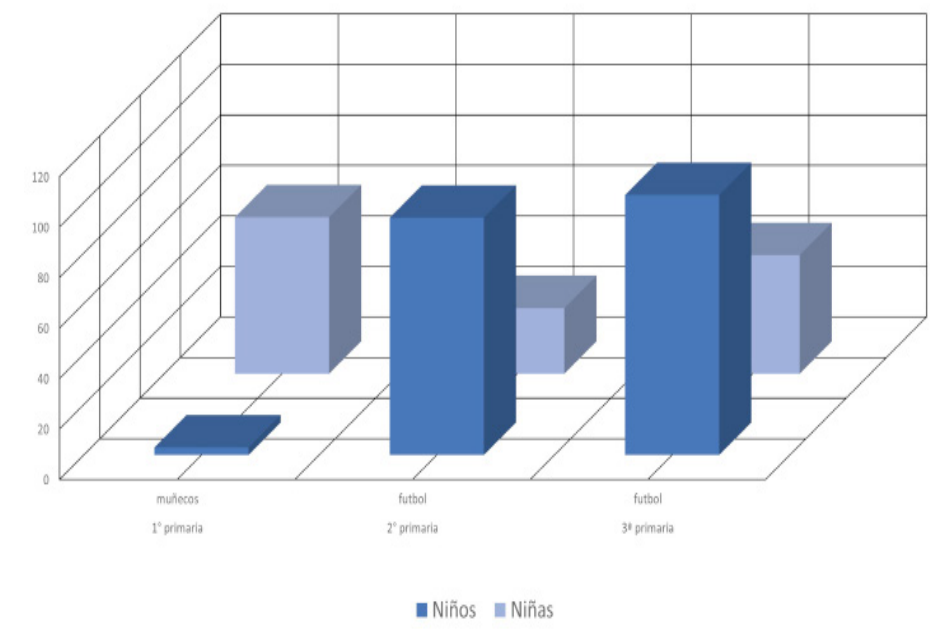

Figura 2.

Frecuencias de juegos en barrio o casa de niños y niñas

Por el tipo de escuela, oficial y privada, las niñas de primer grado juegan más a las muñecas que los niños. Los del segundo grado juegan atrapadas y los de escuelas privadas presentan los puntajes más altos. Los niños del tercer grado y de primaria pública juegan al futbol y obtienen las puntuaciones más altas de los tres grados (ver figura 3).

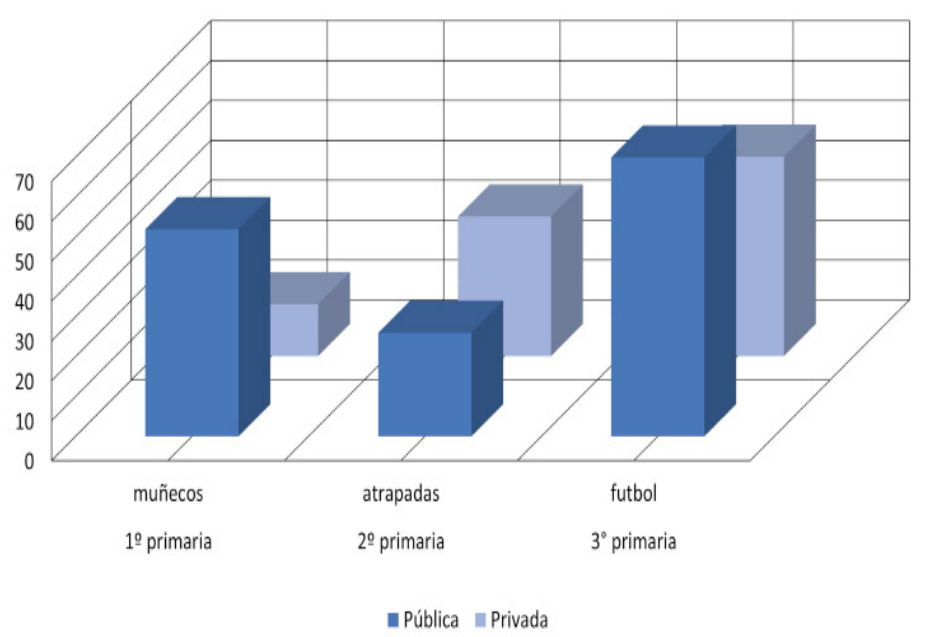

Figura 3.

Frecuencias de juegos en el barrio o casa de niños

Por último, en el juego favorito, en el primer grado las más altas frecuencias las presentan los niños que juegan futbol y las niñas que juegan a las muñecas, en el segundo grado los niños juegan futbol y las niñas escondidas, y en el tercer grado ocurre lo mismo que en 
el grado anterior, la diferencia estriba en que aumenta la cantidad de niñas que juegan futbol, pero no en la misma proporción que los niños; en términos generales más niñas juegan escondidas que futbol, y en los niños es contrario el efecto, juegan más futbol que escondidas (ver figura 4).

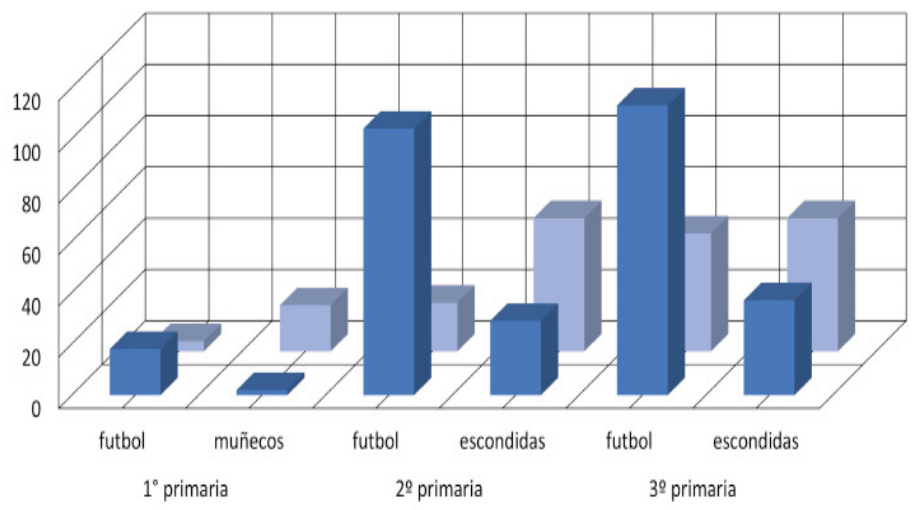

Masculino $\quad$ Femenino

Figura 4.

Frecuencias de juegos favoritos de niños y niñas

En la figura 5 observamos, una vez más, los importantes incrementos en la frecuencia de los niños que juegan el futbol; en tanto, en las niñas el juego escondidas es el que más les gusta. El futbol se juega más en escuelas públicas, y el juego de escondidas se juega más en escuelas privadas.

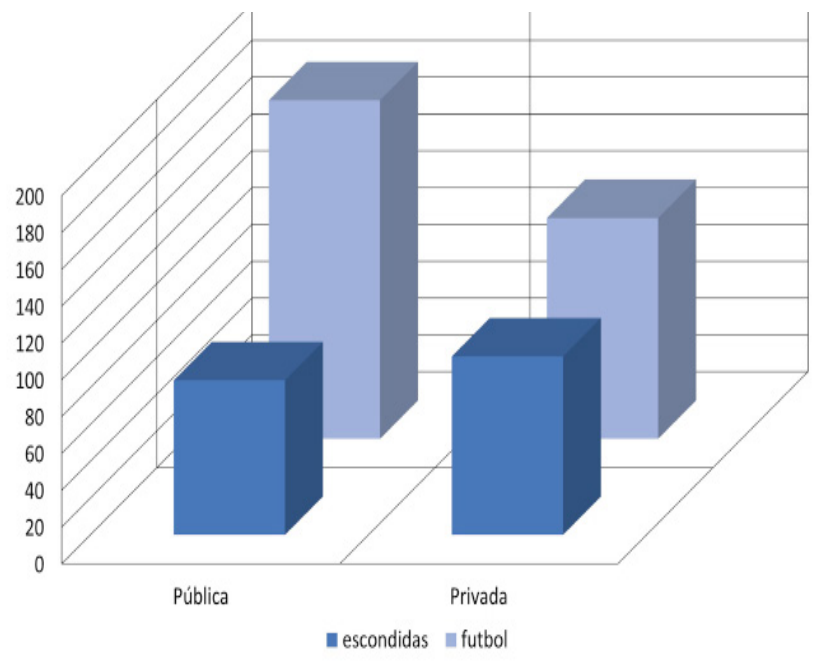

Figura 5.

Frecuencias de juegos favoritos y tipos de escuelas

\section{DiCUSIÓN Y CONCLUSIONES}

La muestra de los niños que se eligió en este estudio corresponde a la etapa del subperiodo preoperatorio y la etapa del pensamiento concreto; los niños en el subestadio preoperatorio o intuitivo tienen habilidades para representar una acción mediante el pensamiento y el lenguaje, su pensamiento es prelógico, se afianza la función simbólica; el niño pasa de la inteligencia prácti$\mathrm{ca}$, basada en el ejercicio, a la inteligencia representativa, en esquemas de acción interna y simbólica, a través de los signos, símbolos, imágenes, conceptos, etc. Esta nueva capacidad de crear y combinar representaciones abre varias posibilidades, ya que libera el pensamiento del aquí y ahora propio de la inteligencia práctica.

En el pensamiento preoperatorio los niños hacen juicios basados en las apariencias percibidas inmediatas, tienden a centrarse en un solo rasgo, tienen dificultad para ponerse en el lugar del otro -egocentrismo-, atienden los estados presentes y no a las transformaciones (centración temporal). Manejan un pensamiento irreversible, no son sensibles a las contradicciones, no logran realizar una correcta lectura de la experiencia de lo que está sucediendo frente a ellos, tienen escasa capacidad de reflexión sobre la propia acción y carecen de toma de conciencia (Delval, 1994).

El siguiente estadio al que pertenecen los niños de la muestra es el pensamiento concreto, hacia los 7 años aproximadamente se inicia una serie de cambios importantes en el pensamiento del niño, éste alcanza formas de organización de su conducta que son superiores a las anteriores etapas. Comienzan los niños a realizar razonamientos cognoscitivos, acciones mentales que permiten llevar a cabo operaciones como la adición, la sustracción, etc.

El niño ya enfoca los problemas desde una perspectiva radicalmente distinta, puede aplicar todo un sistema de operaciones potenciales sobre los aspectos específicos. El niño opera desde la lógica y desde la matemática, desligándose paulatinamente de la percepción hacia los objetos.

El pensamiento del niño se descentra, se vuelve reversible y se consolida la noción de conservación (la posibilidad de mantener estables determinadas propiedades del objeto, como la cantidad, peso, volumen, longitud, superficie, etc.). El establecimiento de invariantes, la conservación de la sustancia, de los líquidos, de las clasificaciones, la seriación y la noción del número (Delval, 1991).

En este estadio del pensamiento concreto la función semiótica prevalecerá y el pensamiento del niño adquiere una característica muy importante, el poder 
representar lo que hasta ahora sólo ha realizado en acciones. Es un mecanismo muy rápido y móvil que puede recordar el pasado, representar el presente y anticipar el futuro en un acto organizado y breve (Flavell, 1979).

Los juegos que practican los niños escolares en los diferentes espacios reflejan sus preferencias o necesidades, éstas pudiesen ser causadas por su nivel de desarrollo y el tipo de juego, por el género o bien por los contextos que propician esos juegos.

Por las probables razones que fuesen, existen importantes argumentos para estudiar el juego. Linaza (2013) menciona que los niños y niñas logran en la práctica de sus juegos diversas adquisiciones sociales, es entonces responsabilidad de los adultos que les rodean fomentarlos, así como proporcionar a los niños los espacios y oportunidades para jugar puesto que es la mejor manera de atender a su derecho a jugar.

El juego como estrategia didáctica se comprende cuando primero los niños juegan de manera cooperativa y creativamente para propiciar el desarrollo de habilidades cognitivas y socio-afectivas. Por este motivo, el juego se considera como una fuente de aprendizaje, el cual origina y desarrolla la imaginación y la creatividad, además de ser una vía de expresión y control emocional.

El trabajo de Piaget (1971) constituye una referencia básica en la toma de conciencia de las reglas, a partir de la cual otros autores han podido constatar la relevancia de ésta en la práctica social del juego.

Bruner (2012) enriquece y amplía esta perspectiva sobre la construcción del conocimiento y la asocia directamente con la propia educación infantil, el autor menciona que la educación: "no consiste sólo en llegar a dominar un contenido sino que también consiste en conocer y comprender... Sí, estoy afirmando que deberíamos cultivar en nuestros escolares una sensibilidad epistemológica apropiada, una conciencia de los procesos implícitos en aprender y en pensar y no únicamente en los productos finales de dichos procesos a los que llamamos currículo... Considero de extremada urgencia que cultivemos este sentido de lo posible en nuestras prácticas educativas" (p. 13).

El autor también aboga por estimular a los niños a explorar el propio proceso de conocer y de comprender, dirigiendo su atención no sólo al producto de conocer sino al proceso de conocer, y recalca que sus actitudes en el juego nos deben convencer de que esto es posible. Con la aparición de los juegos de ficción se produce un cambio profundo en la actividad infantil. Objetos y gestos se transforman para simbolizar otros que no están presentes, lo fundamental no son las acciones sobre los objetos, sino lo que estos y aquellas representan.
Una de las muchas capacidades importantes del desarrollo emocional y que quizás no se valora lo suficiente, es el desarrollo del juego de fantasía, por medio de éste los niños expresan sentimientos trascendentales dentro de un contexto controlado, y el repetir experiencias en variadas formas simbólicas, pueden llegar y enfrentar el conflicto y los sentimientos dolorosos; desde este punto de vista, Sroufe (2000) afirma que el juego de fantasía es emocional.

Entre otras funciones del juego se encuentran las emociones que son fuente de información relevante de los sentimientos y miedos que los niños están viviendo (Navarro, 2002). En concordancia con los aspectos externos de la regulación emocional, se demostró que el juego de los niños con historias de seguridad emocional exhibieron una riqueza en la calidad de sus fantasías, estos niños no solamente mostraron mayor dedicación sino también mayor flexibilidad y complejidad en su juego, y con una gama más amplia de temas emocionales (Roscenberg, 1984, como se citó en Sroufe, 2000).

En cada uno de los contextos en que jugaron los niños se estimularon diferentes juegos, por ejemplo, en el colegio se aprovechó la gran cantidad de niños que hay propiciando que las niñas y niños se integren con sus compañeros y jueguen conjuntamente a las atrapadas o a las escondidas. Pero también hay que considerar el hecho de que los niños más pequeños disfrutan tipos de juegos sensoriomotores, en donde lo importante es el ejercicio, las sensaciones y el placer de correr para atrapar a alguien.

Las elecciones hechas por los niños están sujetas a razones de género, claramente el futbol tiene un gran valor y fuerte impacto sobre los niños, éste es el juego más practicado por ellos. Aquí la regla es importante como lo es el trabajo en grupo, su coordinación e integración son fundamentales, así como el respeto entre los jugadores.

Las personas admiran a alguien destacado en el mundo deportivo por lo que, en ocasiones, principalmente los niños, tratarán de emular las habilidades con la pelota para demostrar su admiración y posiblemente desearán ser futbolistas para alcanzar la fama y el elevado status socioeconómico y extraordinaria popularidad que gozan las grandes figuras futbolísticas. Tal pareciera, aunque es arriesgado decirlo, que es el juego preferido de los varones en este país, pero también es cierto que los medios de comunicación hoy en día ejercen la mayor influencia sobre este deporte en la opinión de la gente.

En el caso de las niñas más pequeñas que eligieron mayormente jugar con las muñecas, este tipo de juego simbólico, específicamente juego de roles, ejercen una influencia definitiva sobre todo en niñas menores que eligen aquellos juegos que hacen referencia a la identificación 
de roles de los miembros de la familia como la mamá, el papá, por los patrones de comportamiento femeninos y masculinos que tienen un componente familiar y social.

Las niñas más pequeñas prefieren jugar a las muñecas, esto podría deberse a que en esta etapa del desarroIlo infantil se encuentran en la identificación de personajes familiares, este juego eminentemente simbólico es ejemplo de ello. Su función principal es la realización de deseos y la resolución de conflictos y esto supone un cambio cualitativo muy importante. También es usual que los niños se identifiquen con roles propios de su género, en el caso de las niñas sería el cuidar a niños, por lo que prefieren jugar a la muñeca.

Winnicott (1981) afirma que existe la necesidad imperiosa de un juego de rol social e individual libre para desarrollar una identidad humana, tal fue el caso de las niñas que juegan a las muñecas para emular patrones de crianza de la madre, cumpliendo de alguna manera esa necesidad de identificarse con sus familiares con quienes mantienen fuertes vínculos emocionales.

Freud (1925, como se citó en Garzarelli, 2005) decía que cada niño, en su juego, se comporta como un escritor creativo, ya que crea su propio mundo y reordena las cosas de éste, en una nueva forma más agradable.

Este estudio exploratorio puede ser una opción importante para diseñar un sistema de enseñanza aprendizaje basado en las elecciones de los juegos de los niños y niñas mexicanas en estos grados para motivarlos a participar de forma más activa y mejorar su aprendizaje. Además, promover una mejor socialización para disminuir en lo posible o quizás hasta erradicar la violencia entre niños y niñas. 


\section{Referencias}

Aizencang, N. (2005). Jugar, aprender y enseñar. Relaciones que potencian los aprendizajes escolares. Buenos Aires: Manantial.

Bernabeu, N. \& Goldstein, A. (2009). Creatividad y Aprendizaje. El juego como herramienta pedagógica. Madrid: Narcea.

Bisquerra, A. R. (2000). Consideraciones sobre la clasificación de las emociones [Monografías]. En: Educación Emocional y Bienestar. Escuela Española. Barcelona España.

Bruner J. (1989). Acción pensamiento y lenguaje. Compilación de José Luis Linaza. Madrid: Alianza.

Bruner, J. S. (2012). What Psychology Should Study? International Journal of Educational Psychology, 1 (1), 5-13.

D Agostino, M. \& Raimbalult A. M. (2004). El juego en el desarrollo del niño. Revista Psicología. Enero-Febrero.

De la Torre, S. (2006). Teoría interactiva y psicosocial de la creatividad. En: S. de la Torre \& V. Violant (Eds.). Comprender y evaluar la creatividad. Un recurso para mejorar la enseñanza. Málaga: Ediciones Aljibe.

Delval, J. (1991). Crecer y pensar. La construcción del conocimiento en la escuela. Barcelona: Paidós.

Delval, J. (1994). El desarrollo humano. México: Siglo XXI de España Editores.

Elkonin, D. B. (1980). Psicología del Juego. Madrid, España: Pablo del Rio.

Flavell, J. H. (1979). La psicología Evolutiva de Jean Piaget. Buenos Aires, Argentina: Paidós.

Garzarelli, J. C. (2005). El Juego, soporte del Deporte. Parte I. Revista Electrónica Psicología del Deporte. Buenos Aires.
Gómez, R. B. (1991). Juegos infantiles tradicionales en la provincia de Mendoza. Editorial de la Facultad de Filosofía y Letras, Universidad Nacional de Cuyo, Argentina.

Hugges, F. P. (2006). El juego su importancia en el desarrollo psicológico del niño y del adolescente. México: Trillas.

Huizinga, J. (1970). Homo Ludens: a study of the play element in culture. New York: J. Harper Editions.

Linaza, J. (2013). El Juego es un derecho y una necesidad de la infancia. Revista Bordón, 65 (1), 103-117.

Navarro, A. V. (2002). El afán de jugar. Teoría y práctica de los juegos motores. México: Manual Moderno.

Piaget, J. (1971). El criterio moral en el niño. Barcelona: Fontanella.

Piaget, J. (1992). La formación del símbolo en el niño. México: Fondo de Cultura Económica.

Piaget, J. (1995). Seis estudios de psicología. Colombia: Ed. Labor.

Ramírez, D. R. N. (2004). La inteligencia emocional en estudiantes universitarios. Revista Electrónica de Psicología. UNAM FES Iztacala, 7 (1).

Sroufe, A. (2000). Desarrollo Emocional. La organización de la vida emocional en los primeros años. Gran Bretaña: Ed. Oxford.

Tejerina, I. (1999). El juego dramático en la Educación Primaria, Textos de Didáctica de la Lengua y la Literatura [Monografía]. Teatro y juego dramático, 19, 33-90.

Vygotsky, L. S. (1979). El desarrollo de los procesos psicológicos superiores. Barcelona, España: Grijalbo.

Vygotsky, L. S. (1970). Psicología del arte. Barcelona: Editorial Barral.

West, J. (2000). Terapia de Juego Centrada en el Niño. México: Manual Moderno.

Winnicott, D. (1981). El proceso de maduración en el niño. Barcelona: Laia. 


\section{Meta-Análisis del Artículo}

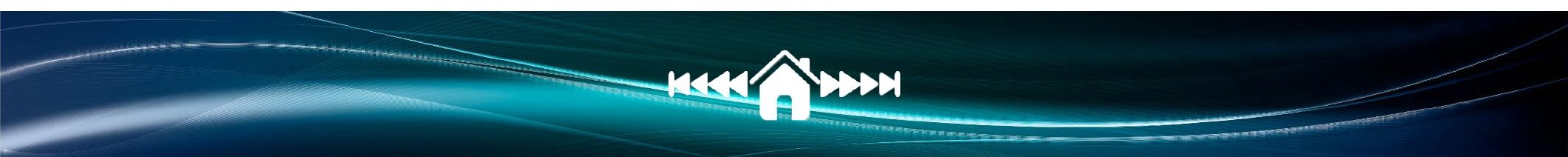


Revista Digital Internacional de Psicología y Ciencia Social $\mid$ Vol. $2 \mid$ Núm. $1 \mid$ Enero-Junio 2016 | ISSN 1405-5802

\section{Dimensión Cuantitativa}

\section{Perfil de Evaluación entre pares}
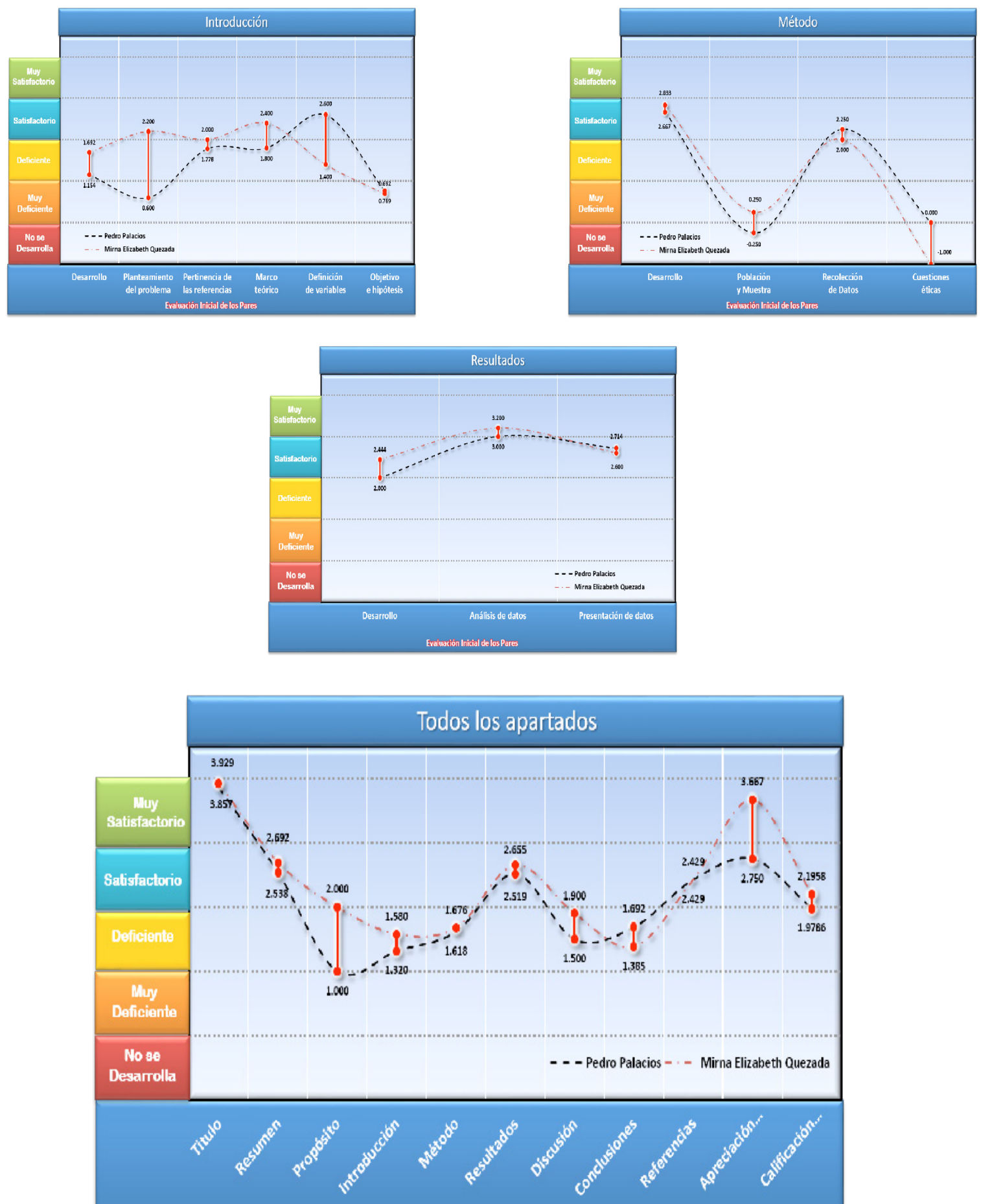

Evaluación Inicial de lo5 Pares 


\section{Índice de Concordancia}

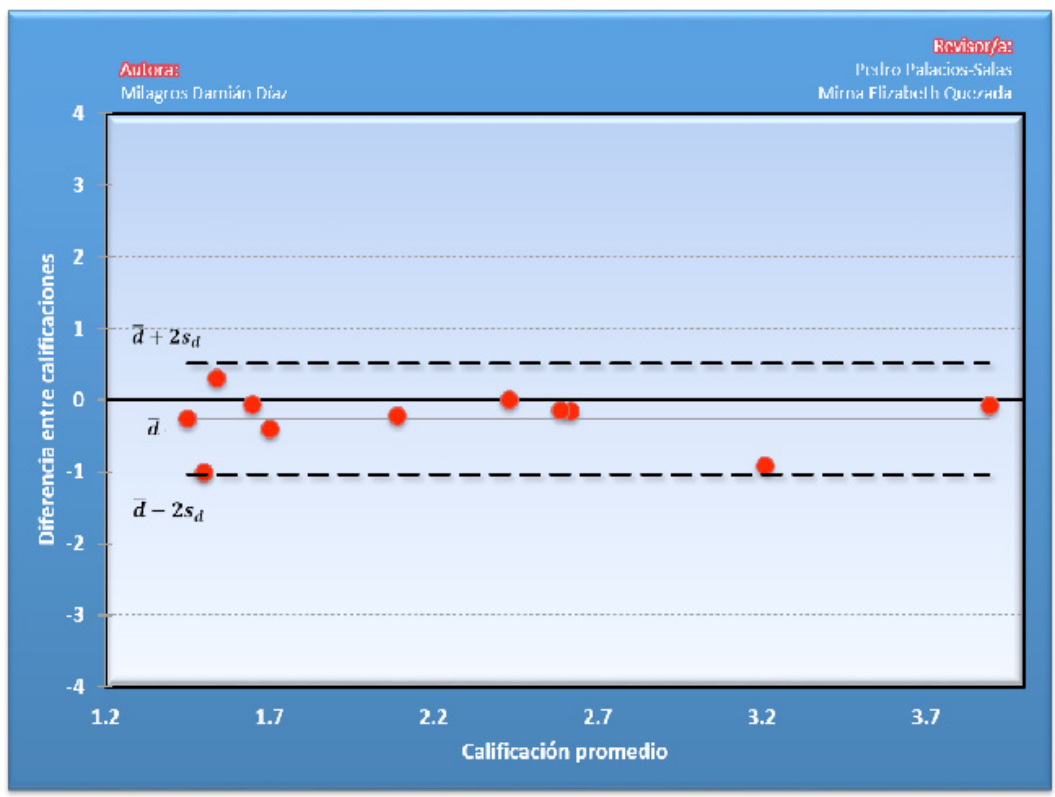

Índice de Acuerdo

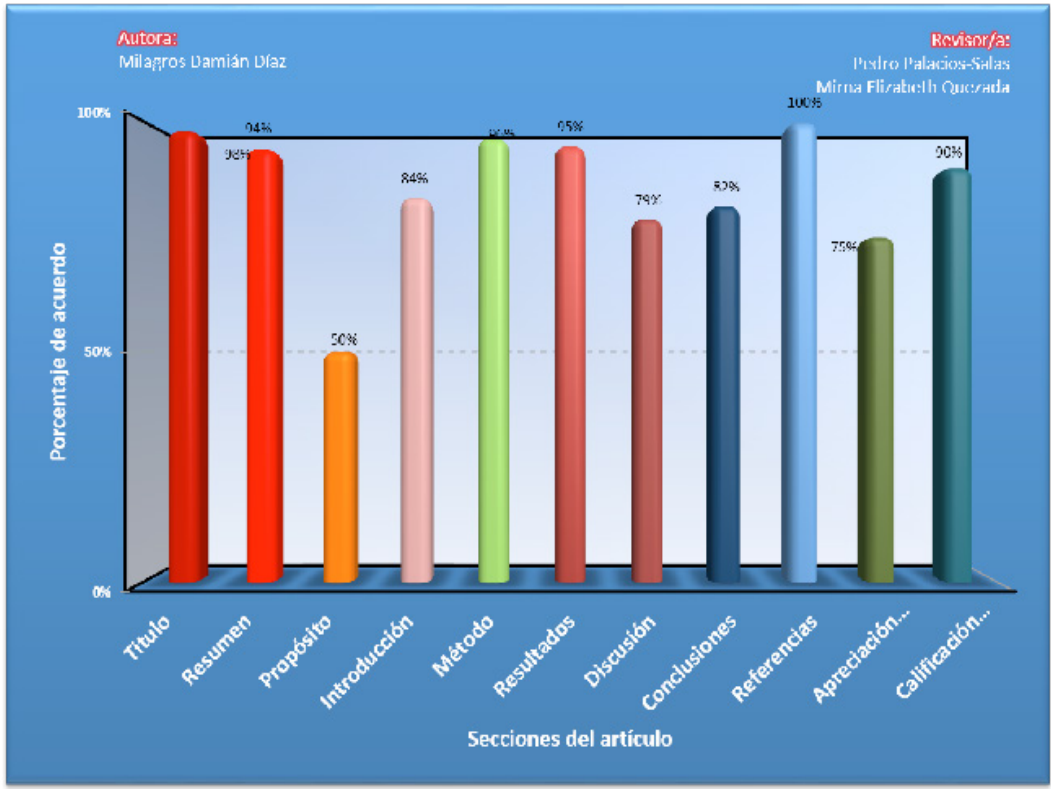




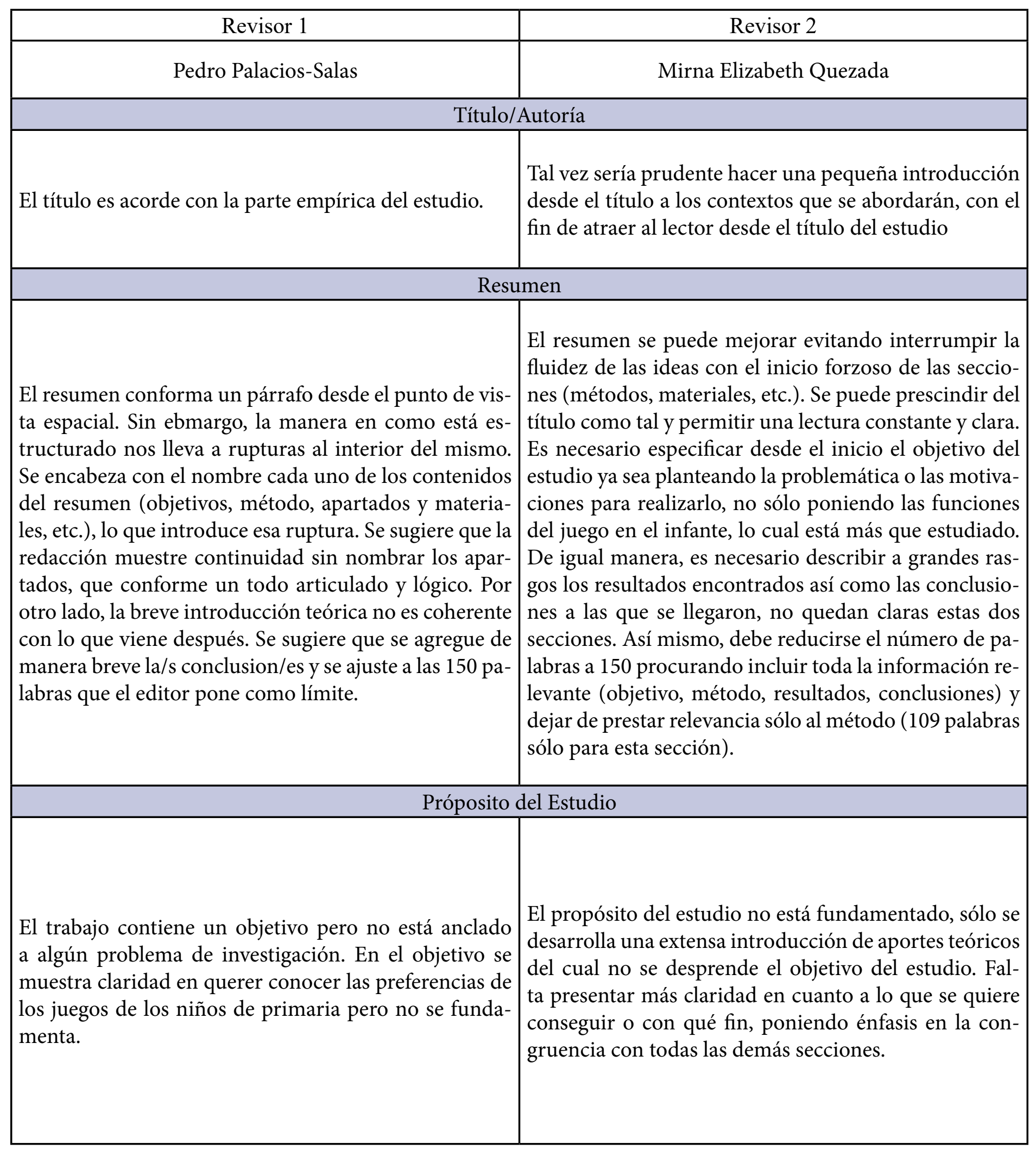




\begin{tabular}{|c|c|}
\hline Revisor 1 & Revisor 2 \\
\hline \multicolumn{2}{|c|}{ Introducción } \\
\hline $\begin{array}{l}\text { En la introducción se hace un repaso general del juego, } \\
\text { de sus implicaciones en el desarrollo psicológico y en las } \\
\text { emociones. La revisión sobre el juego y desarrollo psi- } \\
\text { cológico se basa principalmente en las aportaciones de } \\
\text { Piaget y Vygotski, sin dejar en claro qué de esos aportes } \\
\text { se asumen y cuáles son fundamentales para el presente } \\
\text { estudio (la elección de juegos en distintos contextos). } \\
\text { No fue posible identificar el punto de partida teórico de } \\
\text { esta investigación. Tampoco fue posible identificar qué } \\
\text { aporte teórico o metodológico se hace con este estudio. } \\
\text { El contenido de la introducción no arropa el objetivo, } \\
\text { no se explicita la relación e implicación de los plantea- } \\
\text { mientos teóricos descritos con el objetivo. El manuscri- } \\
\text { to también carece de un planteamiento del problema, } \\
\text { lo que dificulta la valoración de la pertinencia de la re- } \\
\text { visión teórica. Se sugiere que se adecue la introducción } \\
\text { al propósito del presente estudio, que la introducción } \\
\text { sea el punto de partida teórico del estudio. }\end{array}$ & $\begin{array}{l}\text { No queda clara la congruencia del propósito del estu- } \\
\text { dio con la introducción presentada, no se lee en toda la } \\
\text { introducción una sóla idea que de pie al planteamiento } \\
\text { del problema, y se cae recurrentemente en citas exten- } \\
\text { sas de dos o tres autores. La introducción debiera ser el } \\
\text { respaldo de todo el trabajo, pero hay tanto vagaje teóri- } \\
\text { co que no queda claro de donde se desprende la nece- } \\
\text { sidad de estudiar la elección de los juegos. Es necesario } \\
\text { responder a esta necesidad y presentar con congruen- } \\
\text { cia guiada del por qué se hace lo que se hace y el cómo } \\
\text { se hace. }\end{array}$ \\
\hline \multicolumn{2}{|c|}{ Método } \\
\hline $\begin{array}{l}\text { Se sugiere que se indiquen los criterios usados para } \\
\text { realizar la selección de los participantes. También se- } \\
\text { ría conveniente que se agregue el dato de la cantidad } \\
\text { de niños por edad. Surge la duda de si el asistir a una } \\
\text { escuela privada u oficial refleja el nivel socioeconómico } \\
\text { de los participantes. No se describe el procedimiento } \\
\text { de recogida de datos y si se pidió el consentimiento in- } \\
\text { formado de los participantes o a los padres de los niños } \\
\text { más pequeños. }\end{array}$ & $\begin{array}{l}\text { Faltaría especificar los criterios de selección de mues- } \\
\text { tra, así como más detalles demográficos que describan } \\
\text { la complejidad de la muestra. También faltaría justi- } \\
\text { ficar el por qué se toma como predictor del nivel so- } \\
\text { cioeconómico la pertenencia a una escuela privada o } \\
\text { pública cuando no siempre es así. En este caso es mejor } \\
\text { sólo mencionar tipo de escuela, y tal vez no el "nivel } \\
\text { socioeconómico". También faltaría especificar como se } \\
\text { protegieron los datos de los participantes. }\end{array}$ \\
\hline \multicolumn{2}{|c|}{ Resultados } \\
\hline $\begin{array}{l}\text { Al inicio de la sección de los resultados se hace referen- } \\
\text { cia a "la gran diversidad de juegos". Sería interesante } \\
\text { conocer esa gran diversidad, añadiendo el dato de la } \\
\text { cantidad de juegos que conforman esa diversidad. }\end{array}$ & $\begin{array}{l}\text { No existen como tal las hipótesis en este estudio, lo que } \\
\text { es una clara deficiencia, ya que aunque sea un estudio } \\
\text { exploratorio la teoría debiera aportarnos una tendencia } \\
\text { a los resultados, y con mayor razón debido a la nume- } \\
\text { rosa cantidad de estudios que giran en torno al tema. } \\
\text { Las figuras son congruentes con el texto, sólo faltaría tal } \\
\text { vez presentar una variedad más amplia dependiendo el } \\
\text { número de variables a graficar. Habría que cambiar el } \\
\text { tiempo de redacción a pretérito como lo determina los } \\
\text { lineamientos de la revista. }\end{array}$ \\
\hline
\end{tabular}




\begin{tabular}{|c|c|}
\hline Revisor 1 & Revisor 2 \\
\hline \multicolumn{2}{|c|}{ Discusión } \\
\hline $\begin{array}{l}\text { En el apartado de discusión se hace evidente que la in- } \\
\text { troducción parece no tener una relación muy estrecha } \\
\text { con los resultados: Prácticamente no se retoma nada } \\
\text { de la introducción en la discusión. Ésta se organiza re- } \\
\text { saltando la importancia del contexto y género, princi- } \\
\text { palmente. Sería conveniente que en la introducción se } \\
\text { les diera también importancia a estos elementos. Es lla- } \\
\text { mativo que en la discusión, las aseveraciones no están } \\
\text { sustentadas teóricamente, ni relacionadas con otros es- } \\
\text { tudios. De hecho, en la discusión no hay ninguna cita. } \\
\text { La manera como se discute el dato, da la impresión que } \\
\text { es una interpretación más intuitiva. }\end{array}$ & $\begin{array}{l}\text { En la discusión se repiten los resultados con ciertas } \\
\text { opiniones no fundamentadas por estudios previos, por } \\
\text { lo tanto, es un área de oportunidad para el manuscrito. } \\
\text { Esta situación se podría corregir si desde la introduc- } \\
\text { ción se abordaran investigaciones previas y concretas } \\
\text { sobre el problema de estudio (lo cual es inexistente). } \\
\text { Por otra parte, en ningún apartado se habla de las li- } \\
\text { mitaciones del estudio, y debiera ser esta sección la que } \\
\text { de pauta a esta información para enriquecer los traba- } \\
\text { jos posteriores. Aún así, la secuencia de esta sección es } \\
\text { apropiada. }\end{array}$ \\
\hline \multicolumn{2}{|c|}{ Conclusiones } \\
\hline $\begin{array}{l}\text { Incluir las contribuciones del estudio, así como sus de- } \\
\text { ficiencias y precisar las conclusiones. }\end{array}$ & $\begin{array}{l}\text { Mismo comentario de la sección anterior. Especificar } \\
\text { las limitaciones del estudio además de profundizar en } \\
\text { el último planteamiento de seguimiento que se men- } \\
\text { ciona. }\end{array}$ \\
\hline \multicolumn{2}{|c|}{ Referencias } \\
\hline Apegarse al estilo APA en la lista de referencias. & $\begin{array}{l}\text { La mayoría de las referencias no están actualizadas. Se } \\
\text { sigue hablando de estudios de hace más de } 40 \text { años, } \\
\text { cuando deberían abordarse continuación de esos es- } \\
\text { tudios para refutar, proponer o complementar con los } \\
\text { resultados a los que se llegan, son muy pocos los que } \\
\text { aportan sustento al objetivo mismo del manuscrito. Es } \\
\text { una de las principales deficiencias y es notable desde la } \\
\text { primer lectura a la introducción. }\end{array}$ \\
\hline
\end{tabular}


Artículo Empirírico | Los juegos elegidos por niños en el colegio, en la casa y el favorito.| Damián-Díaz, MM.

Historia del Proceso EDITORIAL 